\title{
Fundamentals of Tandem Mass Spectrometry: A Dynamics Study of Simple C-C Bond Cleavage in Collision-Activated Dissociation of Polyatomic Ions at Low Energy
}

\author{
Anil K. Shukla, Kuangnan Qian, Stephen Anderson, \\ and Jean H. Futrell \\ Department of Chemistry and Biochemistry, University of Delaware, Newark, Delaware, USA
}

The loss of methyl radical in collision-activated dissociation (CAD) of acetone and propane molecular ions has been studied at low energy using a tandem hybrid mass spectrometer. Although the two processes are very similar chemically and energetically, very different dynamical features are observed. Acetyl ions from acetone ion are predominantly backwardscattered, with intensity maxima lying inside and outside the elastic scattering circle, confirming our previous observation that electronically excited states are important in low-energy acetone CAD. Ethyl ions from propane ion show a forward-scattered peak maximum at a nonzero scattering angle, which is consistent with generally accepted models for vibrational excitation and redistribution of energy before dissociation. Both processes demonstrate that CAD at low energy proceeds via small-impact-parameter collisions with momentum transfer. Comparison of the present results with higher energy CAD dynamics studies and earlier work leads to some tentative general conclusions about energy transfer in these processes. (J Am Soc Mass Spectrom 1990, 1, 6-15)

$\mathrm{O}$ ur understanding of collision-activated dissociation (CAD) reactions in organic and analytical mass spectrometry has grown enormously over the past two decades in parallel with the dramatic increase in tandem mass spectrometry (MS/MS) applications and the increased availability of commercial instruments of various configurations [1]. Fundamental investigations of these processes initially emphasized experimental and theoretical studies of small and medium-sized molecules in the kilovolt energy range and were later extended to lower energies. A substantial body of information now exists on collisional energy transfer and energy release in the dissociation of activated ions. These studies have facilitated the development of generalized mechanisms for the activation and dissociation steps $[1,2]$ in MS/MS.

Although many studies of kinetics and mechanisms of small-ion decompositions and more qualitative mechanistic studies of large polyatomic ions have been carried out, very little information is available on the dynamics (i.e., velocity vector distributions for product ions originating at the collision center) of CAD processes. The database is particularly limited for polyatomic ions. Herman et al. [3] reported a dynamic study of the CAD of methane and propane molecular ions at low energy and demonstrated that the

Address reprint requests to Jean $\mathrm{I}$. Futrell, Department of Chemistry and Biochemistry, University of Delaware, Newark, DE 19716. observed dynamics are consistent with the generally accepted model of vibrational excitation of the polyatomic ion followed by its unimolecular decomposition. This interpretation assumes statistical redistribution of energy and rapid dissociation of ions that are activated by energy deposition slightly above the thermochemical threshold. Stated otherwise, this study supports the view that $\mathrm{CAD}$ of polyatomic ions can be adequately rationalized using the concepts of the quasi-equilibrium theory (QET) of mass spectra [4]-at least at low collision energy. A correlation of energy deposition with scattering angle was also demonstrated, suggesting the possibility of a quantitative approach to angle-resolved mass spectrometry.

We recently investigated acetone ion $\operatorname{CAD}[5,6]$ and found a qualitatively different behavior from that reported for methane and propane [3]. These results challenge to some extent the hypothesis that mechanisms of CAD in the low-energy regime can be generalized. These contrasting results prompted the present investigation of the $\mathrm{CAD}$ reaction dynamics of the propane and acetone molecular ions carried out under identical laboratory conditions on the same instrument.

In this paper we compare the following reactions at about $1.15 \mathrm{eV}$ collision energy with $\mathrm{He}$ collision gas:

$$
\begin{aligned}
\mathrm{CH}_{3} \mathrm{COCH}_{3}^{+} & \rightarrow \mathrm{CH}_{3} \mathrm{CO}^{+}+\mathrm{CH}_{3} \\
\mathrm{CH}_{3} \mathrm{CH}_{2} \mathrm{CH}_{3}^{+-} & \rightarrow \mathrm{CH}_{3} \mathrm{CH}_{2}^{+}+\mathrm{CH}_{3}
\end{aligned}
$$


These reactions have obvious similarities. Their thermochemical thresholds for dissociation are $0.65 \mathrm{eV}$ [7] and $0.95 \mathrm{eV}$ [8], respectively. Both reactions proceed directly without rearrangement before dissociation, consistent with the hypothesis of a loose transition state [9] for both ionic fragmentations. Further, these dissociations are not competitive with unimolecular metastable decay processes; the metastable process for both ions is the rearrangement elimination of methane, which proceeds via a tight transition state. Both reactions 1 and 2 proceed with negligible reverse activation energy and small kinetic shifts [10]. Both have been investigated by using a variety of experimental techniques, and there is a good general understanding of these fragmentation processes.

Despite these many similarities, the detailed kinetics of the unimolecular decay of excited acetone and propane ions are markedly different. The propane ion has served for many years as an archetypical example of ions whose dissociation processes are welldescribed by the QET of mass spectra [11, 12]. For acetone, on the other hand, it has been demonstrated in carcful studies by Lifshitz et al. [13, 14], and by others [15-18] that the metastable decay and CAD of the acetone ion are bimodal and cannot be explained by the QET, that is, it is an archetypical example of a non-QET molecular ion. The detailed reasons for this difference are not completely understood, and nonQET behavior by polyatomic ions is a current research topic in ion chemistry. Nevertheless, it is now well established that the acetone molecular ion is charactertzed by a band of electronically isolated levels that do not communicate efficiently with the ground electronic state $[6,19]$. This violates one of the fundamental principles of the QET, and the decay processes of this ion may be expected to exhibit either subtle or profound differences from its QET-type analogue, the propane molecular ion.

The present experiments were carried out with the new tandem hybrid mass spectrometer that we constructed especially for the purpose of investigating the dynamics of CAD reactions [20]. The first- and secondstage instruments are commercial mass spectrometers, the collision region is a supersonic molecular jet, and the ion optics and energy and angular deflection components are of our own design. The results will be discussed and presented in the center-of-mass (CM) framework. The $\mathrm{CM}$ reference frame is the most appropriate format in which to present dynamics data and describe the fundamentals of energy transfer and reaction mechanisms [21].

\section{Experimental}

The instrument used for the present study has been described in detail elsewhere [20], and only a brief description is given here. Ions generated by electron impact (EI) ionization ( $70 \mathrm{eV}$ electrons) and accelerated to high energy ( $3 \mathrm{keV}$ ) by a VG $7070 \mathrm{E}$ double-focusing mass spectrometer are decelerated to the desired low energy at the collision center by a series of cylindrical and rectangular tube lenses. At the collision center the low-energy ion beam intersects a vertically moving molecular beam of helium atoms formed by supersonic expansion through a $100-\mu \mathrm{m}$ nozzle. The energy and mass of fragment ions are analyzed by using a hemispherical energy analyzer and quadrupole mass filter, respectively. The detector assembly is rotated about the intersection zone of the ion and neutral beams to determine energy and intensity distributions as a function of scattering angle.

The energy distributions of the fragment ions at various angles are transformed into velocity contour diagrams in the $\mathrm{CM}$ reference frame as described elsewhere [20]. Data are presented in the form of contour maps drawn by joining points of equal intensity. The region on the right-hand side of the diagram between the ion velocity vector and the normal to the $\mathrm{CM}$ is referred to as forward scattering (CM angles $0-\pi / 2$, referenced to the ion beam), and the region on the left, as backward scattering (CM angles $\pi / 2-\pi$, referenced to the ion beam and following the neutral beam path).

\section{Results and Discussion}

As noted in the Introduction, very few MS/MS experiments have analyzed collisional activation and dissociation processes as a problem in reactive scattering dynamics. Accordingly we preface our discussion of results with a brief introduction to the construction and interpretation of scattering contour diagrams. A more detailed discussion of key points in scattering theory and a compilation of the relevant equations for transforming data between laboratory and $\mathrm{CM}$ reference frames can be found in Chapters 2 and 8 of ref 21. Transformation procedures specific to the apparatus used in the present research are given in ref 5 .

The logical starting point for discussing scattering processes is to consider elastic scattering as a baseline example. In collision chamber MS/MS, the neutral target molecules have only random thermal motion and can be considered to be at rest in the laboratory frame. The fast projectile ion strikes the neutral species and, in purely elastic scattering, transfers kinetic energy to it. For the simplest possible case of hard-sphere collisions, a glancing (tangential) collision transfers no energy, while a line-of-centers (zero impact parameter) collision transfers the maximum possible kinetic energy to its partner. All impact parameters between zero and the hard-sphere collision diameter ${ }_{s} R_{c}=R_{1}+R_{2}$, result in angular scattering between $0^{\circ}$ and $180^{\circ}$. Each scattering angle is associated with the exchange of a specific amount of kinetic energy, increasing smoothly from zero to the maximum allowed by conservation of energy and momentum. For all centrosymmetric potentials, elastic scattering trajectories are a function of only the masses of the two particles, their initial kinetic energies, their interaction potential, and the impact pa- 
rameter for the collision. Chapter 2 of ref 21 provides an instructive discussion of structureless classical trajectories for both hard-sphere and Morse potentials. The latter example illustrates the influence of attractive potential wells on elastic scattering. Potentials that more realistically describe the interaction of ions with neutral species are also discussed.

As any amateur pool player can attest, the results of elastic collisions in the laboratory frame present a wonderful variety of possibilities. In sharp contrast, the CM frame presents a very monotonous result. The particles approach the CM origin, or collision center, and then retreat with the same kinetic energy. The only change allowed by the conservation laws is rotation of the relative velocity vector about the collision center. The postcollision distribution of allowed velocities (momenta) is a sphere, and any planar section (e.g., the plane swept by a detector) is a circle with the $\mathrm{CM}$ as its origin. This circle is called the elastic scattering circle (ESC) in the discussion that follows. By factoring out the extraneous forward motion of the collision partners in a particular laboratory setting, the laboratory-to-CM transformation not only produces a dramatic simplification in the presentation of results but also presents data in a format suitable for discussing both energy exchange and angular scattering properties for inelastic collisions.

Because elastic scattering involves no exchange of kinetic and internal energy, it is of no direct interest in CAD. However, it is the boundary zone between translationally endothermic and translationally exothermic processes. In general, CAD processes are inelastic scattering events in which translational energy is converted into internal energy to drive the dissociation process. The amount by which a translationally endothermic process is displaced inside the elastic scattering circle in the contour diagrams that follow is used to measure the extent of conversion of kinetic energy into internal energy.

The construction of scattering contour diagrams is illustrated in Figure 1, which shows the laboratory velocity vectors of the reactant ion and neutral collider. Since our experiment involves a supersonic beam of neutral particles, the neutral beam-shown vertically in the figure-is not at rest as in a collision chamber experiment. Both the ion and neutral beams are sufficiently narrow and monoenergetic that they can be represented as vectors in Figure 1. The measured beam energies, laboratory intersection angle, and masses allow us to calculate the $\mathrm{CM}$ velocity and the $\mathrm{CM}$ relative velocities shown schematically in this figure. The angular rotation of the detector, set to record the mass of the product and measure its kinetic energy distribution, provides several slices of three-dimensional (intensity, energy, angle) data from which the scattering contour diagram can be constructed.

Figure 1 illustrates schematically how the data are both collected and reduced to $\mathrm{CM}$ coordinates. The detector (comprising a hemispherical energy analyzer, quadrupole mass filter, and pulse-counting multiplier) is pivoted about the intersection point of the ion and neutral beams. At each angle, the kinetic energy of the detected ion is scanned as shown schematically by the $I(m, \theta, v)$ insert. The reactant ion and neutral and product ion energies are converted to velocities, $I(v)$; for a series of laboratory angles. The laboratory velocity of the $\mathrm{CM}$ is calculated from the conservation of linear momentum constraint, and product ion velocities with respect to the CM are calculated. Mathematically this involves vector subtraction of the $\mathrm{CM}$ velocity from the observed laboratory velocities of the detected product. Figure 1 illustrates schematically the geometric construction for this vector subtraction for a selected scattering angle. The $\mathrm{CM}$ velocity vectors of the primary ion, $u_{p}$, and the neutral collider, $u_{\mathrm{N}}$, are shown explicitly to conform to this vector subtraction. The ESC that we use as a starting point in our discussion of energy transfer is also shown.

Figure 2 elaborates the construction of a hypothetical diagram for two assumed dynamics models for acetone ion CAD. The baseline of Figure 2 is the hypotenuse of the CM scattering triangle that we have just discussed in Figure 1. In this postcollision diagram the reactant acetone ion, $\mathrm{Ac}^{+}$, retreats from the $\mathrm{CM}$ toward the right and the neutral atom retreats from the CM toward the left. These two directions in the CM frame define the forward-scattering (direction of primary ion beam) and back-scattering (direction of primary neutral beam) hemispheres, respectively. The endpoints of the $\mathrm{CM}$ ion and neutral vectors match their laboratory frame counterparts, as shown in Figure 1. After the collision, the excited ion and neutral collider recoil from the CM. Model (a) illustrates forward scattering of the excited ion, while model (b) is back-scattered. The neutral atom velocity vectors for models (a) and (b) recoil from the CM with momenta equal and opposite to those of the excited acetone molecular ions. The subsequent dissociation of the activated acetone ions into acetyl ions (and methyl radicals) gives us the observable flux of CAD products for constructing scattering diagrams.

Before discussing these models further we must confront the unfortunate fact illustrated in Figure 2 that there is a fundamental indeterminacy in discussing the reaction dynamics of CAD processes-namely, that two particles collide and a minimum of three leave the scattering center. For all two-particle problems, only three vectors-reactant ion, reactant neutral species, and product ion-need be measured to establish the scattering properties of the system uniquely. However, in CAD, only the ion product is measured, and the scattering angles and velocities of the two neutral species can have all values allowed by conservation of energy and angular momentum. Consequently, the scattering diagram does not provide a unique determination of the energetics and angular properties of CAD reactions.

The escape from this dilemma in the interpretation 


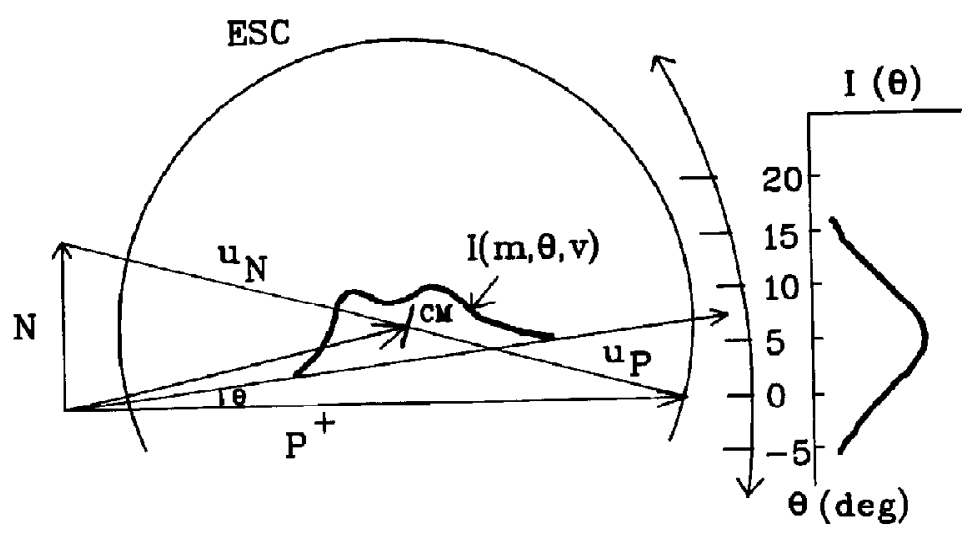

Figure 1. Schematic illustration of the scanning MS/MS method for mapping reactive scattering into the $\mathrm{CM}$ reference frame. The vectors $\mathbf{N}$ and $\mathbf{P}^{+}$are the measured laboratory vectors for the supersonic jet molecular beam $(\mathrm{N})$ and primary ion $\left(\mathrm{P}^{+}\right)$. The detector mass spectrometer is set to observe the product ion mass at a given laboratory angle $\theta$, and the energy distribution and total intensity per unit time are recorded. Energy is converted to velocity, and the data are plotted as $I(m$, $\theta, v)$. When referenced to the $C M$, the resulting plot is the $C M$ contour diagram for the reaction investigated. The CM velocities of the reactant ion, $u_{\mathrm{p}}$, and neutral collider, $u_{\mathrm{N}}$, are also shown. The experiment is repeated at several angles to map as much of the total reaction sphere as possible.

of CAD scattering dynamics is the key assumption that there is a time delay between the excitation process and the decomposition of the excited ion. We think that this assumption is very well founded, especially for polyatomic ions, as the time delay need only be long enough for the neutral collider to escape the potential field of the ion. For typical collisions this will be less than $10^{-13} \mathrm{~s}$. For the example of the rather low energy collision of acetone ions with helium at 1.6 $\mathrm{eV}$ to be discussed later, the relative velocity of the primary ion and neutral is about $1.5 \times 10^{5} \mathrm{~cm} / \mathrm{s}$. As- suming an interaction length of $1.5 \times 10^{-8} \mathrm{~cm}$ leads to a collision time of $10^{-13} \mathrm{~s}$. Decomposition involves a minimum of one and typically thousands to millions of vibrational motions of the nuclei. Dissociation lifetimes are therefore estimated to be much longer than picoseconds.

Figure 2 depicts the scattering results for two collision mechanisms (a) and (b) that deposit the same internal energy, $\Delta E$, in the acetone ion. The conversion of kinetic energy into internal energy requires that the excited acetone ion velocity fall within the ESC. The

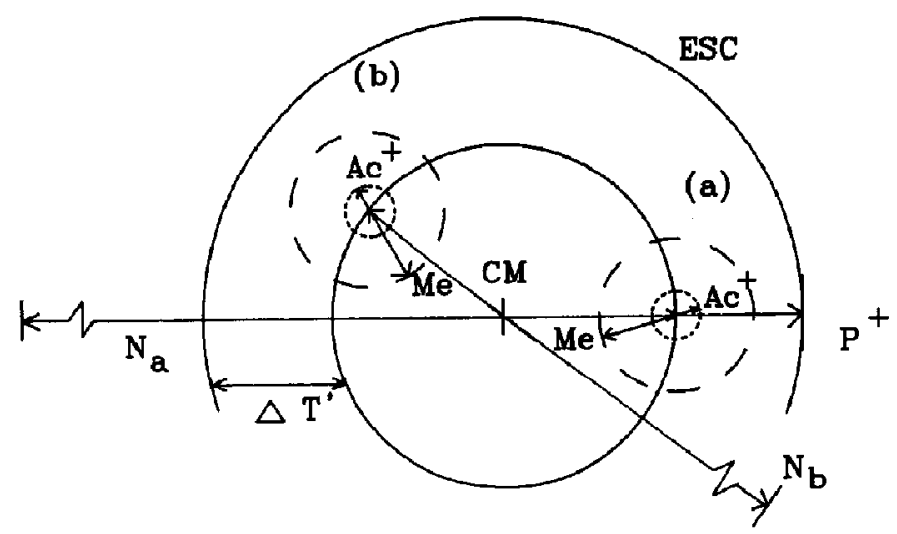

Figure 2. Hypothetical $\mathrm{CM}$ scattering diagram illustrating the reduction of the three-body $\mathrm{CAD}$ problem to a two-body scattering problem. The reactant acetone ion, $\mathrm{P}^{+}$, and neutral collider, $\mathrm{N}$, collide at the $C M$, and the postcollision vectors of elastically scattered $\mathrm{P}^{+}$(ESC) and dissociation products are shown for two dynamical mechanisms. Excitation of the projectile ion by $\Delta E$ through transfer of translational to internal energy exchange, $\Delta T^{\prime}$, is illustrated for a large-impact-parameter glancing collision (forward-scattered, zero scattering angle) and a strongly impulsive, small-impactparameter collision (back-scattered, large scattering angle). The excited acetone ion dissociates isotropically with a small kinetic energy release. Momentum conservation is illustrated by the $\mathrm{CM}$ vectors for the recoiling neutral collider and methyl radical fragments, neither of which is actually detected in these experiments. See discussion in text for details. 
velocity shift corresponds to the energy transferred in the collision, $\Delta T^{\prime}$. Momentum is conserved by the recoil of the helium atom, whose velocity is also appropriately reduced; the postcollision velocities have the same inverse mass ratio as before collision, namely, $\nu_{\mathrm{He}} / v_{\mathrm{Ar}}=m_{\mathrm{Ar}} / m_{\mathrm{He}}$. The velocity vectors of all excitation processes depositing exactly $\Delta E\left(=\Delta T^{\prime}\right.$ by energy conservation) in the acetone ion lie somewhere on the inner circle of Figure 2.

Next we consider the dissociation step. The lifetime of the excited ion is determined by its unimolecular decay kinetics and its energy content. The acetyl ions and methyl radicals separate from their common centroid, which moves with the $\mathrm{CM}$ velocity of their excited acetone ion precursor. Any kinetic energy release is partitioned between the acetyl ion and neutral velocity vectors in the inverse ratio of their masses. Assuming random orientations of the excited acetone precursor, these particles expand in a sphere with the (moving) acetyl ion as a precursor. These relationships with the circles centered on the two relevant centers of mass for models (a) and (b) are depicted in Figure 2 as circles in our sampling plane.

Our two models (a) and (b) assume identical energy depositions and dissociation kinetics. The zeroscattering angle excitation model (a) corresponds dynamically to a large-impact-parameter glancing collision. The back-scattered mechanism shown as model (b) assumes that a small-impact-parameter impulsive collision is involved in the excitation step. For each mechanism, the centroid for the acetyl ion product velocities can be used to deduce $\Delta T^{\prime}$, which defines the energy deposited in the acetone precursor ion by the collision. The width of the distribution is a measure of kinetic energy release in the dissociation step.

Our hypothetical examples illustrate that a $\delta$ function excitation process in which a single, fixed amount of energy is deposited can give precisely predictable $C A D$ reaction dynamics. Since we measured the acetone primary ion and acetyl secondary ion velocity vectors (and also used a well-characterized supersonic beam of neutral species for our second reactant), our experiments can, in principle, be interpreted at the level of detail illustrated in Figure 2. Unfortunately, it is quite unexpected that any polyatomic ion could give as simple a pattern as our models (a) and (b) [22]. Rather than single values for excitation, we anticipate that a distribution of energy transfer mechanisms will result in a distribution of velocity vectors for collisionally excited ions. Similarly, the decomposition step results in some distribution of dissociation dynamics. Together these effects lead to a distribution that may be quite broad, and the most we can learn from our experiment is something about the average dynamical behavior of polyatomic ions.

Our experimental data compare CAD dynamics for acetone and propane molecular ions at nearly $1.6 \mathrm{eV}$ collision energy in Figures 3 and 6 . Contour lines of equal intensity are drawn to show the relative proba-
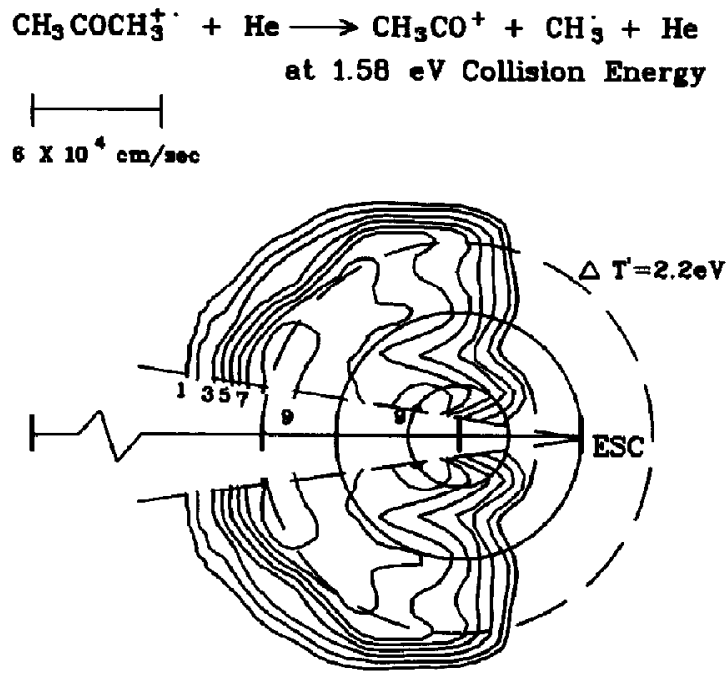

Figure 3. Scattering contour diagram for the CAD of acetone molecular ion to acetyl ion on collision with helium at $1.58 \mathrm{eV}$ collision energy (23.38 eV LAB). Contour lines connect points of equal intensity normalized such that the most intense line corresponds to a relative intensity of 9 and the lowest to 1 . The circle marked ESC represents the elastic scattering circle indicating no energy conversion between translational and internal modes. The circle labeled $\Delta T^{\prime}=2.2 \mathrm{eV}$ circumscribes all velocity vectors of the acetone ion for which the translational energy is increased by $2.2 \mathrm{eV}$. This must arise from the conversion of $2.2 \mathrm{eV}$ internal energy into translation. The innermost circle corresponds to the conversion of $1.3 \mathrm{eV}$ translational energy into internal energy. Dissociation into acetyl ion and methyl radicals requires a minimum of $0.65 \mathrm{eV}$ additional energy to surmount the thermochemical barrier.

bilities of observing acetyl ion and ethyl ion scattered at a particular $C M$ angle with a particular $C M$ velocity. The zero velocity point is the $\mathrm{CM}$ origin, and zero angle is defined by the primary ion direction. Our experimental contour plots use the symmetry property that positive and negative deflection angles are identical to show the fraction of all reaction space mapped by our experiments. A small cone at $180 \pm 10^{\circ}$ is excluded from observation in both figures by geometrical factors of our apparatus that preclude mapping the back-scattered regime completely [5].

Figure 3 is remarkably more structured than we had anticipated for the acetone molecular ion (or for polyatomics generally). Two distinct peaks of nearly equal intensity are observed, both of which lie in the backward-scattered region. Scattering in this sector demonstrates that nearly head-on collisions of small impact parameters dominate the CAD process for acetone ions at this energy.

We first consider the back-scattered peak located outside the ESC. Clearly this peak identifies an exothermic superelastic collisional activation process releasing internal energy into translation. Our recent study of acetone ion CAD at very low collision energies (at and below the thermochemical threshold) demon- 


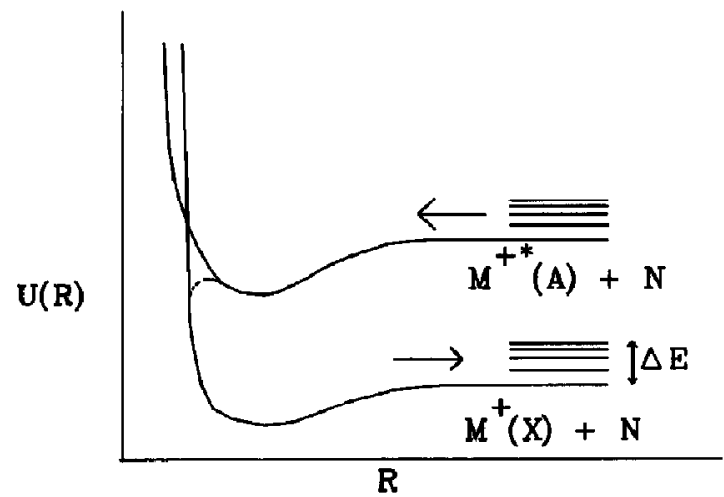

Figure 4. Schematic pseudodiatomic potential energy curves representing the collision path of an excited acetone ion $\mathrm{M}_{1}^{+*}$ with neutral atom $N$ crossing into ground state $M_{1}^{+}$with excess energy release into translation via a curve-crossing mechanism. $\Delta E$ indicates schematically the additional energy required for dissociation to occur from the ground electronic state.

strated that this mechanism involves a long-lived electronic state of the ion [6]. As discussed in detail elsewhere [6], at these very low collision energies, acetone ion in a long-lived electronically excited state collides with helium and releases its excess energy into translation before the dissociation. As discussed in connection with Figure 2 and our models (a) and (b), a relatively tight distribution of acetyl ion velocities implies both a relativcly well defined energy transfer step and a modest distribution of kinetic energy release in the dissociation step. Under these circumstances, energy transfer into the acetone ion can be discussed with confidence. This appears to be the case for the present example.

In contrast with our models, the experimental distribution demonstrates that the dissociating acetone ions giving rise to the peak outside the ESC have much higher translational energy than the incoming ion. This energy-release process is induced by nearly head-on collisions of the ion and neutral. The significant disruption of nuclear positions anticipated for such collisions is the likely triggering event for releasing the stored electronic energy into translation.

Figure 4 indicates, in a very schematic way, our interpretation of how this unexpectedly strong back-scattered electronic-to translational $(E \rightarrow T)$ energy transfer step that initiates $C A D$ of the acetone ion may actually take place. The long-lived excited state population of acetone ions $\left(\mathrm{M}^{+*}\right)$ collides with the neutral atom, $N$, along the upper curve in the figure. At short distances-specifically, at or near the turning point of the trajectory-the system crosses to the lower curve. When the particles separate, the electronic energy of the upper state is released largely as translational energy. Back-scattering implies that this curve-crossing mechanism is accessed mainly by smallimpact-parameter collisions.

The maximum of the peak outside the ESC in Figure 3 lies at about $3.8 \mathrm{eV}$ from the $\mathrm{CM}$. Because the translational energy initially present in the reactants is $1.6 \mathrm{eV}$, the kinetic energy gain via this mechanism is about $2.2 \mathrm{eV}$. Because the thermochemical threshold for reaction 1 is $0.65 \mathrm{eV}$, we can account for the conversion of about $2.9 \mathrm{eV}$ from stored internal energy to translational energy plus the energy required for $\mathrm{CAD}$ proceeding via this mechanism. A similar analysis was put forward in an earlier report that investigated the $\mathrm{CAD}$ of acetone ion at 0.45 and $0.65 \mathrm{eV}$ [6].

It is quite remarkable that an excited state of the acetone ion could remain stable during its passage from the ion source to the collision center even though it had such a large excess of internal energy. However, a number of other experiments also indicated [23, 24] that acetone molecular ion can have excess energy in this range and not decompose. In our earlier paper, we proposed on the basis of similar energetics that this long-lived state is the A state of the acetone ion. We referenced a photoelectron (PE) spectroscopic study that reported that the vertical transition energy is 2.9 $\mathrm{eV}$ [25]. The ground-state energy is lower and of the order of $2.2 \mathrm{eV}$ for the $0-0$ transition [19]. This energy difference matches rather well the kinetic encrgy relcasc we measured in the present experiments and at collision energies of 0.45 and $0.65 \mathrm{eV}$ [6], that is, a constant offset of $\Delta T^{\prime}=2.2 \mathrm{eV}$ separates the maximum from this mechanism from the ESC as the radius of the ESC is changed by changing the collision energy over this range from 0.6 to $1.5 \mathrm{eV}$. Because additional electronic states are located in an overlapping band in the PE spectrum, our suggestion that the A state is responsible for this remarkable CAD mechanism is plausible but may not be definitive.

Microscopic reversibility requires that the reverse pathway for conversion of translational energy into electronic excitation be equally efficient. The endothermic peak in Figure 3 may be evidence for this mechanism. A circle drawn through this inner peak corresponds to $0.3 \mathrm{eV}$ kinetic energy with respect to the $\mathrm{CM}$, or a shift of $1.3 \mathrm{eV}$ from the ESC in this 1.6$\mathrm{eV}$ collision energy experiment. This translational-tointernal energy exchange supplies much more than the $0.65 \mathrm{eV}$ required to surmount the thermochemical barrier. If we add to our energy inventory the $\sim 0.8 \mathrm{eV}$ internal energy that some ground-state acetone ions formed by EI are expected to retain [19, 25], there is just sufficient energy to excite the first excited A state of the acetone molecular ion. Clearly this largely back-scattered, small-impact-parameter "hard core" collision populates highly excited state(s) of the acetone molecular ion. Some, and probably most, are coupled to the acetyl ion dissociation channel and are observed as CAD products. In the $1.6 \mathrm{eV}$ collision energy experiment summarized by Figure 3 , the two pathways-collisional perturbation of the electronic states, which then decay, and the pumping of vibrationally excited ground-state ions to higher (possibly electronically excited) states, which also decay-are coincidentally of nearly equal intensity. 


$$
\begin{aligned}
& \mathrm{CH}_{3} \mathrm{COCH}_{3}^{+\cdot}+\mathrm{Ar} \rightarrow \mathrm{CH}_{3} \mathrm{CO}^{+}+\mathrm{CH}_{3}^{+}+\mathrm{Ar} \\
& \text { at } 300 \mathrm{eV} \text { laboratory }(123 \mathrm{eV} \mathrm{CM})
\end{aligned}
$$
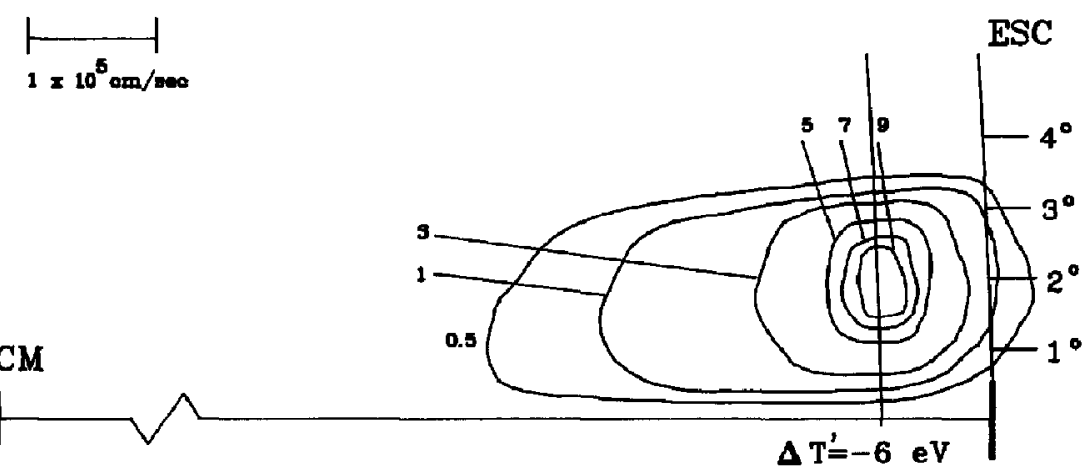

Figure 5. Scattering contour diagram for the CAD of acetone molecular ion to acetyl ion on collision with helium at $123 \mathrm{eV}$ collision energy ( $300 \mathrm{eV} \mathrm{LAB}$ ). Contour lines connect points of equal intensity normalized such taht the most intense line corresponds to a relative intensity of 9 and the lowest to 1. The circle marked ESC represents the elastic scattering circle indicating no energy conversion between translational and internal modes. Only the positive angle deflection portion of the diagram is shown. (Reprinted with permission from ref 5.)

A third dynamic mechanism not energetically accessible at $1.6 \mathrm{eV}$ is also important in acetone ion CAD. Figure 5 is the scattering contour diagram for an acetone ion colliding with argon at $123 \mathrm{eV} \mathrm{CM} \mathrm{col-}$ lision energy. As discussed in detail elsewhere [6], this forward-scattering mechanism is the only one observed over the energy range from tens of electron
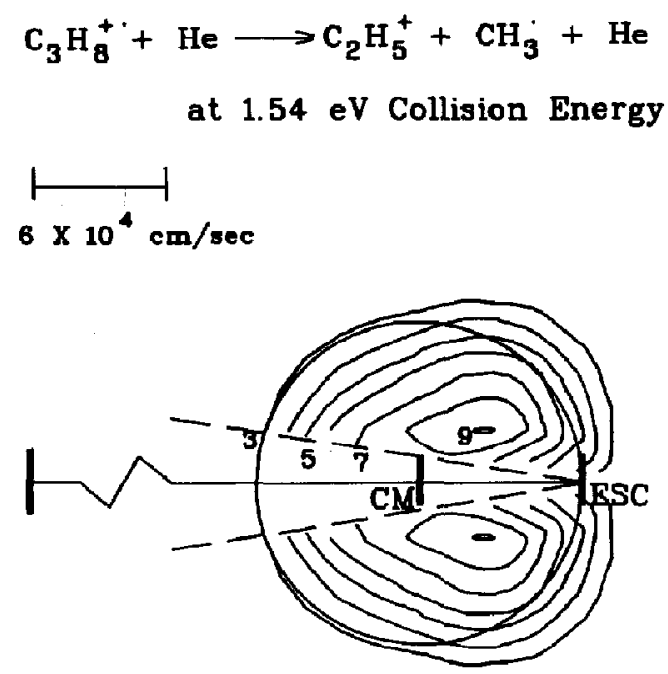

Figure 6. Scattering contour diagram for the CAD of propane molecular ion to ethyl ion on collision with helium at $1.54 \mathrm{eV}$ collision energy (17.8 eV LAB). Contour lines connect points of equal intensity normalized such that the most intense line corresponds to a relative intensity of 9 and the lowest to 1 . The circle marked ESC represents the elastic scattering circle indicating no energy conversion between translational and internal modes. volts to kilovolts. It is highly endothermic, absorbing about $6 \mathrm{eV}$ in the Figure 5 experiment.

The present results are consistent with and bridge our previous CAD studies of the acetone ion $[5,6]$. As the energy is lowered to and below the thermochemical threshold, the relative contribution from the enduthermic charnel decreases dramatically and the superelastic backward-scattered peak completely dominates low-energy CAD. At somewhat higher energy, backward-scattering with a peak maximum within the ESC dominates the CAD process. At the intermediate energy of $1.6 \mathrm{eV}$ reported here, both back-scattered processes are observed with roughly equal intensities. At still higher energy, forward-scattered, highly endothermic scattering becomes the dominant mechanism. The forward-scattering mechanism depicted in Figure 5, which is not observed in low-energy experiments, is the only CAD mechanism observed at kilovolt energies.

Our results for propane ion CAD under experimental conditions nearly identical to those of Figure 3 are summarized in Figure 6 . The $\mathrm{CM}$ velocity contour map for reaction 2 at $1.54 \mathrm{eV}$ collision energy using helium collision gas is shown in Figure 6. In sharp contrast with acetone results, ethyl ions generated from propane ion $\mathrm{CAD}$ are strongly forward-scattered, with the intensity maximum falling within the ESC. This is the expected result for an endothermic inelastic collision process. The maximum is at nonzero scattering angle, as was also observed in an earlier higher energy study [3] using neon as the neutral collider.

The earlier molecular beam study of the dynamics of methane and propane ion CAD used the QET of mass spectra to interpret the dynamics. The conver- 
sion of translational to internal energy (at a given collision energy) was shown to correlate approximately linearly with scattering angle. Different scattering angles for individual CAD channels result from the different energy requirements for competing dissociation processes, as shown most clearly by the QET breakdown graph for the propane molecular ion [11]. A distribution of vibrationally excited ground-state molecular ions are excited by collision to a broad distribution of excited vibronic states of the propane ion. The "hardness" of the collision correlates (via the impact parameter) energy deposition with larger scattering angles. Because ethyl ion formation is a higher energy process than propyl ion or ethylene ion formation, the maximum intensity for ethyl ion formation occurs at a larger scattering angle. This general pattern, also found in our experiments and depicted in Figure 6, is preserved as collision energy is increased.

At higher collision energies, the dynamics of reaction 2 depicted in Figure 6 change only quantitatively $[3,26,27]$. The scattering angles at which the intensity maximum is found closes smoothly to lower values but remains nonzero. This behavior is that which is expected for an impulsive excitation mechanism followed by prompt dissociation with little kinetic release in bond scission. At nearly $500 \mathrm{eV}$ collision energy, the scattering probability contour diagram for propane ions colliding with argon and decomposing by reaction 2 is rather similar to Figure 4 for acetone $[26,27]$. The maximum intensity occurs at $1.8^{\circ}$, and the major difference is that the translational endothermicity is significantly smaller than for acetone.

This invariance of mechanism with collision energy characteristic for reaction 2 is clearly not observed for reaction 1 . We attribute the very different $C A D$ dynamics for the formally similar reactions 1 and 2 to the very different electronic band structures for the two molecular ions. The presence of the carbonyl group profoundly shifts the ground-state ionization level, which may be described for acetone as the removal of a lonepair electron from the oxygen atom. In the PE spectrum, this shows up as an intense peak for the 0-0 transition at $9.7 \mathrm{eV}$. The ground-state vibronic envelope is relatively narrow, and there is a window in the PES spectrum $[19,25]$ from 10.5 to $11.3 \mathrm{eV}$ where no photon absorption occurs. Unfavorable FranckCondon factors render the adiabatic transition to the A state very weak and difficult to observe. It is located at about $11.9 \mathrm{eV}$, and the vertical transition to the $A$ state is found at $12.6 \mathrm{eV}, 2.9 \mathrm{eV}$ above the ground state. Several electronic states overlap in this upper band, and several minima and maxima characterize the shorter wavelength PE spectrum of acetone.

This contrasts strongly with propane, for which the PE spectrum is broad and unstructured [28]. In propane the strong coupling of excited states to the ground state, as required by the fundamental postulates of the QET, leads to the CAD dynamical features we have described. We suggest that the fundamen- tal reasons for the quite different CAD dynamics of acetone is the difference in the electronic structure of the ion. This is manifested most directly in the coarsegrained PES spectrum of acetone and the lack of strong coupling of one or more excited electronic states to the ground state.

Our tentative conclusion from these observations is that acetone ion CAD largely follows an electronic state excitation pathway. This is clearly the case at very low energies [6] and in the present experiments. We suggest that it may also be true at high energy, for which the large energy loss (Figure 5) may result from the excitation of electronic levels higher than $5 \mathrm{eV}$.

These contour diagrams obviously reveal a great deal about mechanisms of collision processes. However, they also can present a misleading picture of the relative importance of different mechanisms. They represent a planar section of the scattering sphere rather than a sampling of the spherical surface. In particular, plotting data in the plane of the reactant beams exaggerate the importance of mechanisms that pile up intensity at the poles of the reaction sphere. Multiplication by the sine of the CM scattering angle corrects for this effect. The total reaction probability, or cross section, is obtained by integration of the differential cross sections derived from our data over spherical angles [20].

A second quantity, elusive in most MS/MS measurements, is the encrgy deposition function. In combination with an appropriate kinetic model, the distribution of internal energy deposited in the collision step defines the rates of subsequent decomposition steps. Within the uncertainties imposed by our low signalto-noise measurements and the assumptions made in interpreting CAD as a stepwise pracess, our experiments permit us to evaluate directly the energy deposition in collisional excitation. This is deduced from the change in translational energy. By energy conservation, the change in kinetic energy, $\Delta T^{\prime}$, is equal to (and opposite in sign) the change in internal energy, $-\Delta U$, for inelastic collision processes. We have already used this concept explicitly in discussing the probable mechanisms revealed by the maxima in Figure 3. The average energy deposition and its distribution are deduced by the appropriate average over energy and scattering angle. The relevant equation is

$$
P\left(T^{\prime}\right) \propto u \int P\left(u_{1}, u_{2}, u_{3}\right) \sin \chi d \chi
$$

where $u$ is the magnitude of the CM product velocity vector; $P\left(u_{1}, u_{2}, u_{3}\right)$ is the probability that products are observed in the space defined by the Cartesian CM coordinates infinitesimally close to $u_{1}, u_{2}$, and $u_{3}$; and $x$ is the CM scattering angle.

Figure 7 is obtained by applying the transformation relation given in eq 3 to the same data set used to construct Figure 3. This figure shows a discernible peak in the energy transfer distribution function for the en- 


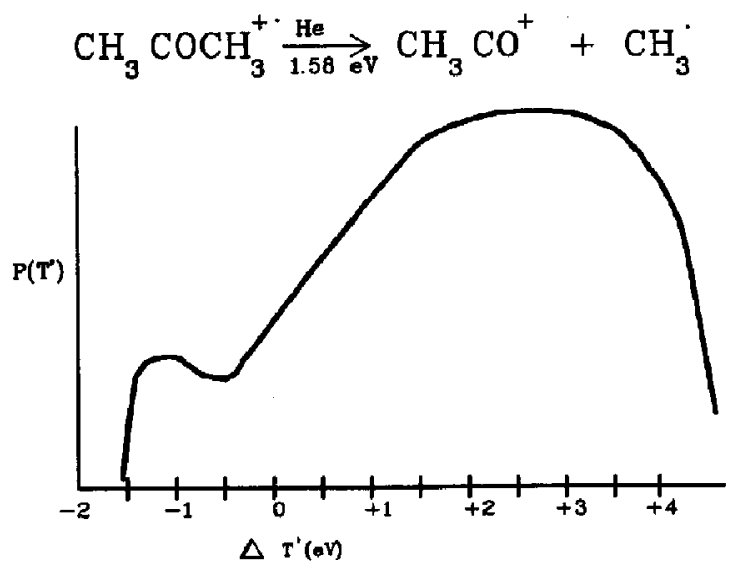

Figure 7. Relative translational energy distribution of acetyl ion at $1.58 \mathrm{eV}$ collision energy expressed as translational exoergicity. Negative $\Delta T^{\prime}$ represents conversion of kinetic energy into internal energy.

dothermic reaction channel and a broad maximum at about $2.9 \mathrm{eV}$ for the exothermic channel. For propane the analogous Figure 8 exhibits no structure in the $P\left(T^{\prime}\right)$ distribution. The mean energy deposition to derive propane ion $\mathrm{CAD}$ is about $0.6 \mathrm{eV}$, a value consistent with the earlier study [3] and with the hypothesis that CAD can be described within the QET framework. Both CAD excitation peaks are broad (and are further broadened by kinetic energy release in the dissociation step), washing out much of the detail present in the contour diagrams, Figures 3 and 6 . A broad range of scattering angles and a broad range of energy depositions promote CAD for both molecular ions. Highly exothermic and highly endothermic processes, and the whole range in between, are part of the $\mathrm{CAD}$ reaction dynamics for acetone. Propane exhibits no exceptional energy transfer features.

We have suggested that the structured scattering patterns for the acetone ion result from its coarsegrained electronic structure. It is therefore disappointing, in some sense, that Figure 7 does not correlate directly with its highly structured PE spectrum. This results, in part, from the integration of CAD processes over the total reaction sphere (momenta of all products, or phase space for the reaction), which largely removes the detail observable in individual angular scans. One pragmatic conclusion, which follows from a comparison of Figures 3 and 7 , is that the angular scanning capability of our tandem spectrometer is its most important characteristic for examining details of CAD reaction mechanisms. The success of angleresolved mass spectrometry in structural CAD studies [29] no doubt relates directly to this same characteristic. Our ability to measure translational energy in the same experiment is also extremely important in that it allows us both to distinguish forward-, side-, and back-scattered processes as qualitative features of $C A D$ mechanisms and to construct detailed scattering contour diagrams.

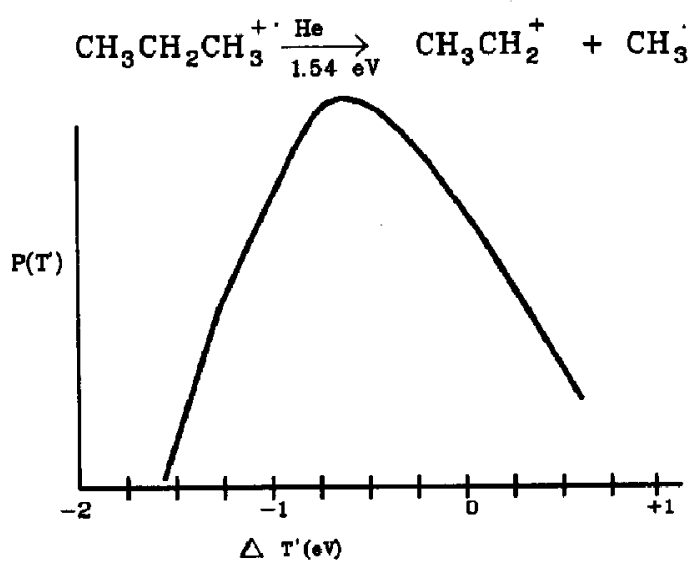

Figure 8. Relative translational energy distribution of ethyl ion at $1.54 \mathrm{eV}$ collision energy expressed as translational exoergicity. Negative $\Delta T^{\prime}$ represents conversion of kinetic energy into internal energy.

A second important conclusion follows from our observation that the $P\left(T^{\prime}\right)$ diagram does not correlate with the PE spectrum (indirectly) or the breakdown graph (directly) for acetone. We infer that CAD excitation does not follow optical selection rules. The range of impact parameters sampled in all collision experiments may emphasize optically allowed transitions at some scattering angles but also populates energy levels not accessed by photon and (high-energy) electron impact. A similar conclusion about CAD excitation mechanisms in general was reached by Cooks et al. [30, 31] from an exhaustive survey of the dependence of ion fragmentation patterns on the mode of excitation.

Recent papers by Cooks et al. [30, 31] focused our attention on the importance of the energy deposition function in determining CAD mechanisms. For some examples, features of the PE spectra of test molecules are found as broadened features in the excitation functions they deduce for CAD. For some systems the excitation function is similar at low and high energy; for others it is different. Our direct measurement of $P\left(T^{\prime}\right)$ for the acetone molecular ion shows that it falls into the latter category. A comparison of Figure 7 with Figure 12 of ref 5 shows that the integrated energy deposition function changes dramatically with collision energy. The shift in $P\left(T^{\prime}\right)$ for the propane molecular ion is much less pronounced over the same energy range $[26,27]$. We speculate that the fundamental factor underlying this difference is that the acetone ion CAD excitation mechanism follows an electronic excitation ladder whereas propane CAD mainly involves vibronic excitation. This speculation clearly identifies CAD studies of non-QET molecular ions as an interesting topic for future research.

\section{Acknowledgments}

We gratefully acknowledge the support of this research by the National Science Foundation, grant no. CHE-8312069. Partial support was provided by the Petroleum Research Fund, grant 
no. 13835-AC6, administered by the American Chemical Society. We also thank Professors Chava Lifshitz and John Eland for helpful discussions of the acetone problem.

\section{References}

1. Busch, K. L.; Glish, G. L.; McLuckey, S. A. Mass Spectrometry/Mass Spectrometry; VCH: New York, 1989. This monograph lists 899 references.

2. Singh, S.; Harris, F. M.; Koyd, R. K.; Beynon, J. H. int. J. Mass Spectrom. Ion Processes 1985, 66, 131.

3. Herman, Z.; Futrell, J. H.; Friedrich, B. Int. J. Mass Spectrom. Ion Processes 1984, 58, 299.

4. Rosenstock, H. M.; Wallenstein, M. G.; Warhaftig, A. L.; Eyring, H. Proc. Natl. Acad. Sci. U.S. A, 1952, 38, 667,

5. Shukla, A. K.; Qian, K.; Howard, S. L.; Anderson, S. G.; Sohlberg, K. W.; Futrell, J. H. Int. J. Mass Spectrom. Ion Processes 1989, 92, 147.

6. Qian, K.; Shukla, A. K.; Howard, S. L., Anderson, S. G.; Futrell, J. H. J. Phys. Chem, 1989, 93, 3889.

7. Traeger, J. C., Hudson, C. E.; McAdoo, D. J. J. Phys. Chem. $1988,92,1519$.

8. Rosenstock, H. M.; Draxl, K.; Steiner, B. W.; Herron, J. T. 1. Phys. Chem. Ref. Data Suppl. 1977, 6, 1.

9. Vestal, M. L. In Fundamental Processes in Radiation Chemistry; Ausloos, P., Ed.; Wiley: New York, 1968; p 59.

10. Levsen, K, Fundamental Aspects of Organic Mass Spectrometry; Verlag Chemie: Weinheim ${ }_{t}$ 1978; pp 35 and 108.

11. Vestal, M.; Futrell, J. H. J. Chem. Phys. 1970, 52, 978.

12. Stockbauer, R.; Inghram, M. G. J. Chem. Phys. 1976, 65, 4081.

13. Lifshitz, C.; Tzidony, E. Int. J. Mass Spectrom. Ion Phys. 1981, 39,181 .

14. Lifshitz, C. I. Phys. Chem. 1983, 87, 2304.
15. McAdoo, D. J.; Witiak, D. N. J. Chem. Soc, Perkin Trans. 2 1981,770 .

16. Turecek, F.; McLafferty, F. W. I. Am. Chem. Sac. 1986, 106, 2525.

17. Stace, A. J.; Shukla, A. K. Int. I. Mass Spectrom. Ion Phys. $1981,37,25$.

18. Johnson, K.; Powis, I.; Danby, C. J. Chem. Phys. 1981, 63, 1.

19. Bombach, R.; Stadelmann, J. P.; Vogt, J. J. Chem. Phys. 1982, 72, 259.

20. Shukla, A. K.; Anderson, S. G,; Howard, S. L.; Sohlberg, K. W.; Futrell, J. H. Int. J. Mass Spectrom. Ion Processes 1988 , 86,61 .

21. Futrell, J. H. In Gaseous Ion Chemistry and Mass Spectrometry; Futrell, J. H., Ed.; Wiley: New York, 1986; pp 25 and 155.

22. Cooks, R. G. In Collision Spectroscopy; Cooks, R. G., Ed.; Plenum; New York, 1978; p 357.

23. Mintz, D. M.; Baer, T. Int. J. Mass Spectrom. Ion Phys. 1977. 25,39 ,

24. Vekey, K.; Brenton, A. G. Rapid Commun. Mass Spectrom. 1988, 2, 156.

25. Brundle, C. R.; Robin, M. B.; Kuebler, N. A.; Basch, H. J. Am. Chem. Soc. 1972, 94, 1451.

26. Anderson, S. G. Ph.D. Thesis, University of Utah, 1990.

27. Anderson, S.; Qian, K.; Shukla, A.; Futrell, J., to be published.

28. Murrell, J. N.; Schmidt, W. J. Chem. Soc., Faraday Trans. 2 $1972,10,1079$.

29. McLuckey, S. A.; Cooks, R. G. In Tandem Mass Spectrometry, McLafferty, F. W., Ed.; Wiley: New York, 1983; p 303.

30. Wysocki, V. H.; Kenttamaa, H. I.; Cooks, R. G. Int. J. Mass Spectrom. Ion Processes 1987, 75, 181.

31. Wysocki, V. H.; Kenttamaa, H. E.; Cooks, R. G. J. Phys. Chem. 1988, 92, 6465. 